\title{
Pustular Psoriasis: A Narrative Review of Recent Developments in Pathophysiology and Therapeutic Options
}

\author{
Alan Menter - Abby S. Van Voorhees · Sylvia Hsu
}

Received: July 28, 2021 / Published online: October 9, 2021

(C) The Author(s) 2021

\begin{abstract}
Pustular psoriasis is an unusual form of psoriasis that frequently presents clinical challenges for dermatologists. The condition presents with pustules on an erythematous background and has two distinct subtypes: localized disease on the palms and soles, called palmoplantar pustulosis (PPP), and generalized pustular psoriasis (GPP). The involvement of the fingers, toes, and nails is defined as a separate localized variant, acrodermatitis continua of Hallopeau, and is now thought to be a subset of PPP. The rarity of pustular psoriasis frequently makes the correct diagnosis problematic. In addition, treatment is limited by a relative lack of evidence-based
\end{abstract}

Supplementary Information The online version contains supplementary material available at https:// doi.org/10.1007/s13555-021-00612-x.

A. Menter $(\square)$

Department of Dermatology, Baylor Scott \& White University, 3900 Junius Street, Suite 145, Dallas, TX 75246, USA

e-mail: amderm@gmail.com

A. S. Van Voorhees

Department of Dermatology, Eastern Virginia

Medical School, Norfolk, VA, USA

e-mail: VanvooAS@evms.edu

S. Hsu

Department of Dermatology, Temple University

School of Medicine, Philadelphia, PA, USA

e-mail: Sylvia.Hsu@tuhs.temple.edu therapeutic options. Current management is often based on existing therapies for standard plaque psoriasis. However, there remains a need for treatments with high, sustained efficacy and a rapid onset of action in pustular psoriasis. Recent advances in understanding of the pathogenesis of pustular psoriasis have provided insights into potential therapies. Treatment of pustular psoriasis is generally determined by the extent and severity of disease, and recent years have seen an increasing use of newer agents, including biologic therapies. Current classes of biologic therapies with US Food and Drug Administration and European Medicines Agency approval for treatment of moderate-to-severe plaque psoriasis in the USA (and elsewhere) include tumor necrosis factor alpha inhibitors (adalimumab, certolizumab pegol, etanercept, infliximab), interleukin (IL)-17 inhibitors (brodalumab, ixekizumab, secukinumab), an IL-12/23 inhibitor (ustekinumab), and IL-23 inhibitors (guselkumab, risankizumab, tildrakizumab). Recently, specific inhibitors of the IL-36 pathway have been evaluated in GPP and PPP, including spesolimab, an IL-36 receptor inhibitor which has shown promising results in GPP. The emerging drugs for pustular psoriasis offer the possibility of rapid and effective treatment with lower toxicities than existing therapies. Further research into agents acting on the IL-36 pathway and other targeted therapies has the potential to transform the future 
treatment of patients with pustular psoriasis. This article reviews the clinical features of PPP and GPP, and current understanding of the genetics and immunopathology of these conditions; it also provides an update on emerging treatments.

\section{PLAIN LANGUAGE SUMMARY}

Pustular psoriasis is a skin condition where people develop small pus-filled blisters on their skin. Pustular psoriasis may affect certain areas of the body, such as the palms and/or the soles. This is called palmoplantar pustulosis (PPP for short). Another type of pustular psoriasis can affect most of the body called generalized pustular psoriasis (GPP for short). Not many people have PPP or GPP. Around 5-12 in every 10,000 people worldwide develop PPP. GPP is even rarer, affecting only 2-7 out of every 1 million people. In addition to being relatively uncommon, these conditions are challenging to treat. This article aims to help doctors who treat skin conditions (dermatologists) to identify and treat people with pustular psoriasis. Currently there is no standard treatment for GPP and PPP in the USA or Europe, but several medicines are approved for treatment of GPP in Japan. Doctors often use treatments that have been shown to work in plaque psoriasis, which is the most common type of psoriasis, to treat people with GPP and PPP. Traditional treatments for PPP and GPP are often not effective. Researchers are working on developing new effective treatments for pustular psoriasis that may work more rapidly and have fewer side effects. These are expected to be available in the next few years.

Keywords: Biologic therapy; Generalized pustular psoriasis; Palmoplantar pustulosis; Psoriasis; Pustular psoriasis

\section{Key Summary Points}

Pustular psoriasis is a form of psoriasis that is rare in clinical practice and therefore can be difficult to recognize

Treatment is limited by a relative lack of evidence-based therapeutic options

This article summarizes recent advances in understanding of the pathogenesis of pustular psoriasis which have led to new potential treatments, including biologic therapies

The emerging therapies for pustular psoriasis offer the possibility of rapid and effective treatment with lower toxicities than existing therapies

\section{DIGITAL FEATURES}

This article is published with digital features, including an infographic, to facilitate understanding of the article. To view digital features for this article go to https://doi.org/10.6084/ m9.figshare.16592891.

\section{INTRODUCTION}

Pustular psoriasis is a form of psoriasis characterized by neutrophil-containing pustules on an erythematous background [1]. It is distinct from the more common plaque psoriasis-not only clinically but also histologically and genetically [2]. Pustular psoriasis is generally considered to be idiopathic, although genetic factors do play a role in disease susceptibility. The condition has two distinct subtypes: localized disease occurring on the palms and soles, known as palmoplantar pustulosis (PPP; also called palmoplantar pustular psoriasis), and generalized pustular psoriasis (GPP). The involvement of the fingers, toes, and nails is defined as a separate localized variant, acrodermatitis 
continua of Hallopeau, which is now thought to be a subset of PPP.

Pustular psoriasis generally follows a relapsing and remitting course, requiring long-term disease management. Precipitating and exacerbating factors include smoking, infections, withdrawal from medications (e.g., systemic corticosteroids, methotrexate, and cyclosporine), and pregnancy. In addition, the use of tumor necrosis factor alpha $(\mathrm{TNF} \alpha)$ inhibitors may occasionally cause paradoxical triggering of immune-mediated skin diseases, including pustular psoriasis [3-5]. At present, there is limited evidence on which to base treatment recommendations for pustular psoriasis, partly due to the less common nature of the condition, and current therapies are based on existing treatments for plaque psoriasis [6, 7]. Nonetheless, recent advances in understanding of the pathogenesis of pustular psoriasis have provided insights into possible targeted therapies, including biologic therapies. This article reviews the clinical features of PPP and GPP, in addition to recent developments in our understanding of the genetics and immunology of the conditions. An update on potential new treatments is also included.

\section{METHODS}

The Medline database was searched via PubMed to retrieve relevant articles on generalized pustular psoriasis, palmoplantar pustulosis, and pustular psoriasis published between 2015 and 2020 (limits: humans, English language). Other relevant literature was obtained on the basis of personal knowledge and experience of the authors. Manual assessment of retrieved references was used as the basis for a narrative overview of the literature.

\section{Compliance with Ethics Guidelines}

This article is based on previously conducted studies and does not contain any new studies with human participants or animals performed by any of the authors.

\section{RESULTS}

\section{Pustular Psoriasis: PPP}

PPP is the most common variant of pustular psoriasis; it has a reported prevalence of $0.05-0.12 \%$ and is significantly more prevalent in Japan than in Western countries $[8,9]$. PPP is a chronic, refractory disease that is associated with major impairment of health-related quality of life, resulting from pain and pruritus [10]. The condition presents with pustules on the palms and soles and is frequently accompanied by erythema, pain, and scaling. The clinical diagnosis of PPP is made in the presence of primary, chronic pustules on the palms, soles, or both (Fig. 1a) [11]. Patients may complain of severe burning pain. When the sterile pustules resolve, there are often brown macules and scaling. The nails may also be affected. The diagnosis of PPP is generally straightforward, although differential diagnoses should be excluded (Fig. 1b).

Smoking and female sex are common risk factors for the development of PPP [12]. Other factors that can precipitate or exacerbate PPP include infections (e.g., tonsillitis and dental infections) and psychological stress [13-15]. PPP can also be precipitated or exacerbated by certain drugs, including TNF inhibitors (infliximab, etanercept, and adalimumab) used for other conditions, such as rheumatoid arthritis or Crohn's disease [16], and interleukin (IL)-17 inhibitors, used for the treatment of psoriasis $[4,5]$. The pathophysiology of this paradoxical drug effect has not been clearly described, but it appears to result from an increased expression of type 1 interferon (IFN) alpha (IFN $\alpha$ ), IFN $\gamma$, and proinflammatory cytokines (IL-36, IL-19, and IL-20) [17]. Recent research suggests that IFN $\alpha$ is produced by CD3/CD4/T-bet-positive type 1 helper T cells [18]. This paradoxical response, which develops in a small minority of patients who receive TNF inhibitor therapy, is likely due to an undetermined genetic predisposition [16]. 
(a)

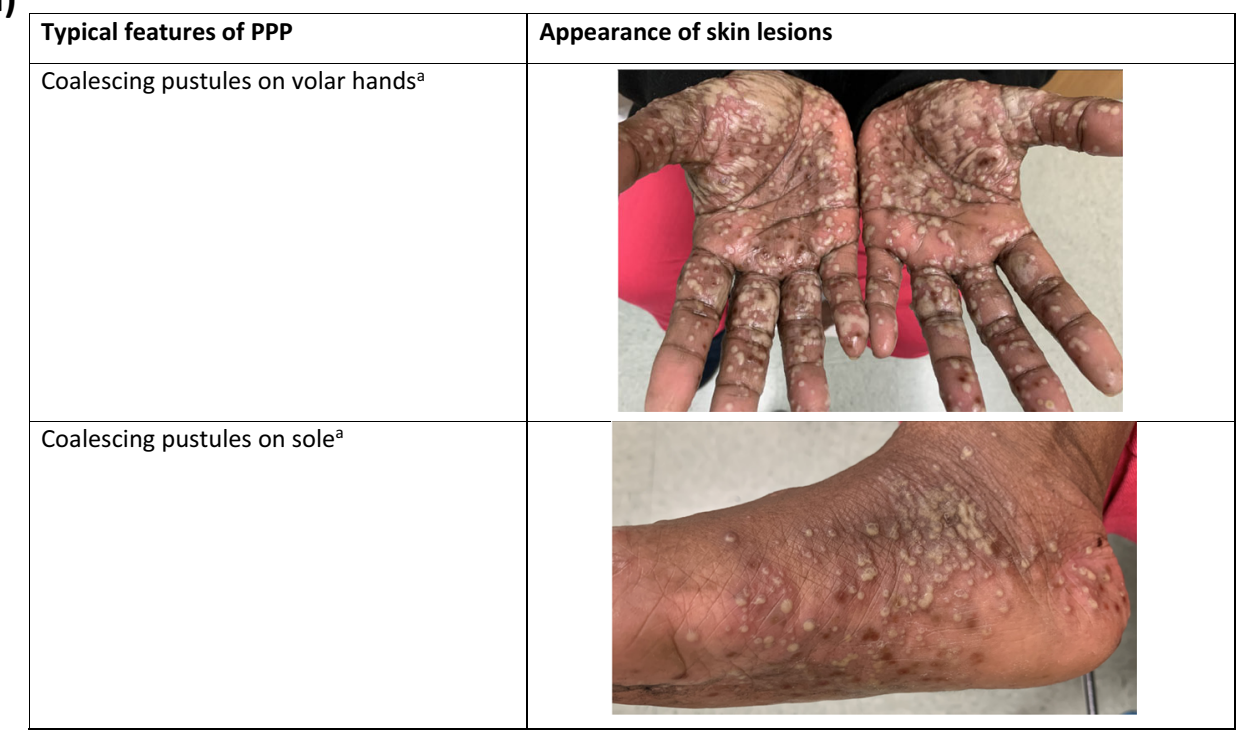

(b)

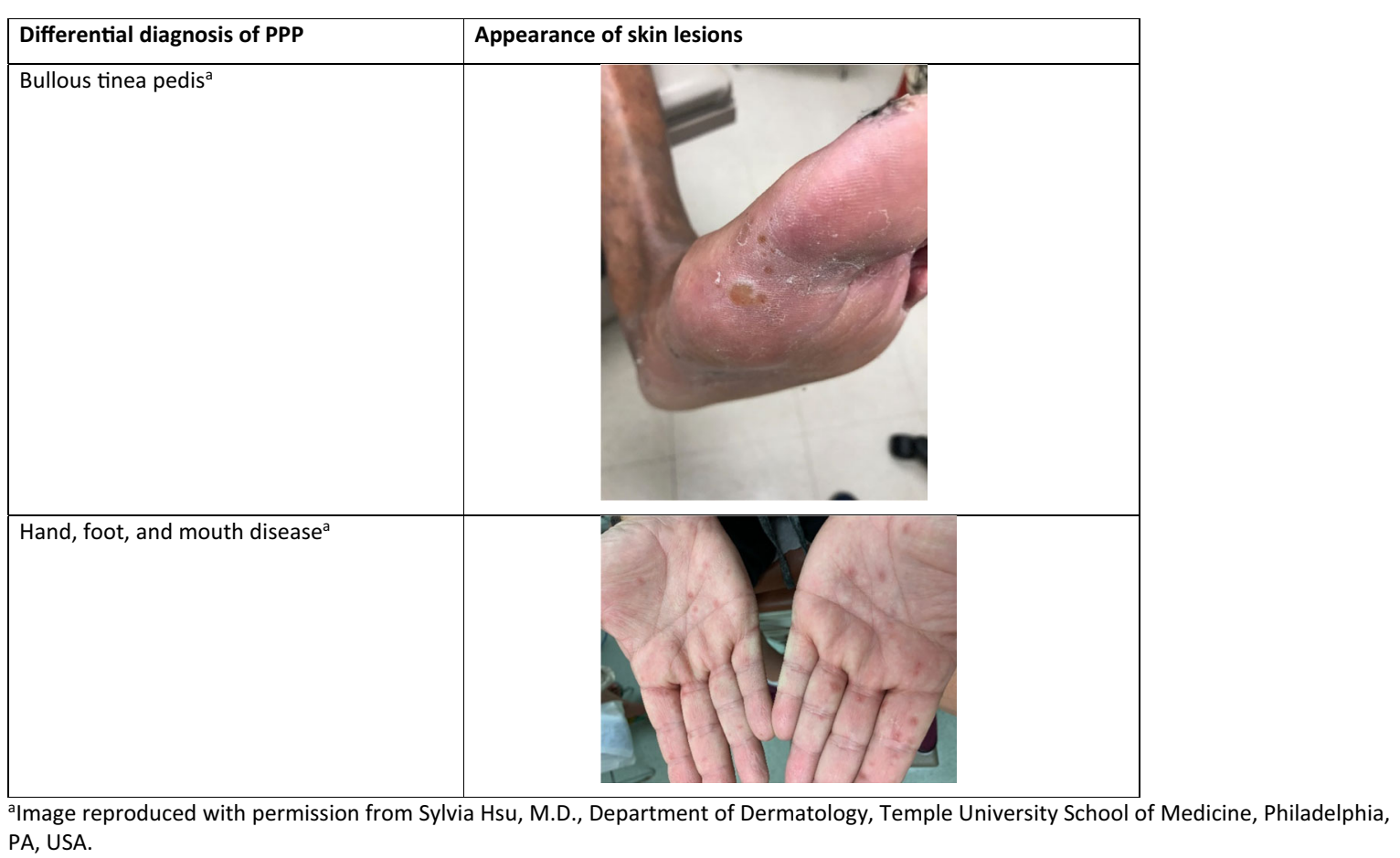

Fig. 1 Clinical features of PPP (a) and differential diagnosis (b). PPP palmoplantar pustulosis

Genetics and Immunopathogenesis of PPP At present, the mechanism underlying the development of PPP is not well understood, and the known genes associated with pustular psoriasis account for only a minority of PPP cases
[12]. However, mutations of the caspase recruitment domain-containing protein 14 (CARD14) gene have been associated with PPP in European patients [19], in addition to AP1S3 gene mutations [12]. Overexpression of sweat 
antimicrobial peptide is thought to underlie the development of PPP [20], and the inflammatory process of PPP likely originates in the acrosyringia, resulting in their destruction [13]. The

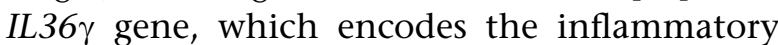
cytokine IL-36 $\gamma$, is upregulated in patients with PPP, indicating that dysregulation of the IL-36 pathway is involved in the development of skin lesions in these patients [21]. Stimulation of the IL-36 receptor results in the activation of

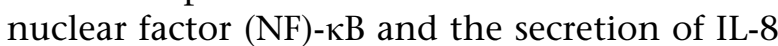
(a chemokine for neutrophils), pro-inflammatory cytokines TNF $\alpha$, IL-1 and IL-23, and an increase in Th-17 activity [20]. In addition to overactivity of the IL-36 pathway, activation of the IL-17 pathway is seen in patients with PPP [22]. IL-17 is produced by T cells and other cell types, including neutrophils and mast cells, contributing to the inflammatory processes underlying PPP, as well as plaque psoriasis [22].

\section{Pustular Psoriasis: GPP}

GPP is a rare skin disease that has an estimated worldwide incidence of $2-7$ per million $[23,24]$. The condition is characterized by widespread eruption of neutrophilic, sterile pustules, which characteristically have surrounding erythema. According to the European Rare and Severe Psoriasis Expert Network definition, GPP can occur with or without systemic inflammation [11]. In contrast, Japanese guidelines for the management of GPP state that GPP is considered to be a systemic inflammatory condition, with patients having abnormal clinical findings associated with inflammation, such as fever and raised levels of C-reactive protein [25]. The clinical course of GPP varies between individual patients, and can follow a relapsing or persistent course. The severity of symptoms can vary with each disease flare and within each individual patient (Fig. 2a) [2]. Patients with GPP require careful management, since severe cases can result in life-threatening complications (e.g., sepsis, cardiorespiratory failure) $[25,26]$. Japanese guidelines for the management and treatment of GPP define four main features of the disease: (1) systemic symptoms (e.g., high fever, fatigue); (2) systemic or widespread pustules; (3) subcorneal pustules with neutrophils histopathologically characterized by Kogoj's spongiform pustules; and (4) the frequent recurrence of these features $[25,27]$. Differential diagnoses of GPP should be excluded when relevant, in particular drug eruptions such as acute generalized exanthematous pustulosis (associated with drugs such as amoxicillin or nonsteroidal anti-inflammatory drugs) [28] (Fig. 2b).

Several factors can precipitate or exacerbate acute GPP. Withdrawal of systemic corticosteroids is a known trigger for GPP flares [29]. Other triggers include pregnancy (GPP in pregnancy was previously also known as impetigo herpetiformis) [30], stress, and TNF $\alpha$ inhibitors $[1,2]$. In addition, infectious respiratory viruses have been identified as trigger factors among a small cohort of patients with various psoriasis subtypes, including GPP [31]. It is possible that the viral infection and associated stimulation of the innate immune system produce a dysregulated immune response that leads to the onset of GPP.

\section{Genetics and Immunopathogenesis of GPP}

The proposed immunopathogenetic mechanisms underlying the development of GPP are complex and have been reviewed by Bachelez et al. [2], Furue et al. [32], and Johnston et al. [33]. The histopathology of GPP is characterized by extensive epidermal infiltration by neutrophils and mononuclear cells, leading to the development of clinically visible, sterile pustules $[1,11]$. Overexpression of IL-36 or a lossof-function mutation of its antagonist, IL-36RA, is thought to play a central role in the pathogenesis of pustular psoriasis [34, 35]. Mutations of the IL36RN gene encoding the IL-36RA are the most frequent genetic abnormality associated with GPP, especially in European and East Asian populations [12], with a reported frequency of IL36RN gene mutation of between $23 \%$ and $61 \%[12,34,36-40]$. The greater frequency of IL-36 mutations in GPP versus PPP is an important indicator that the two conditions are genetically and immunopathologically distinct.

Mutation of the IL36RN gene results in an unstable IL-36RA protein with a reduced affinity 
(a)

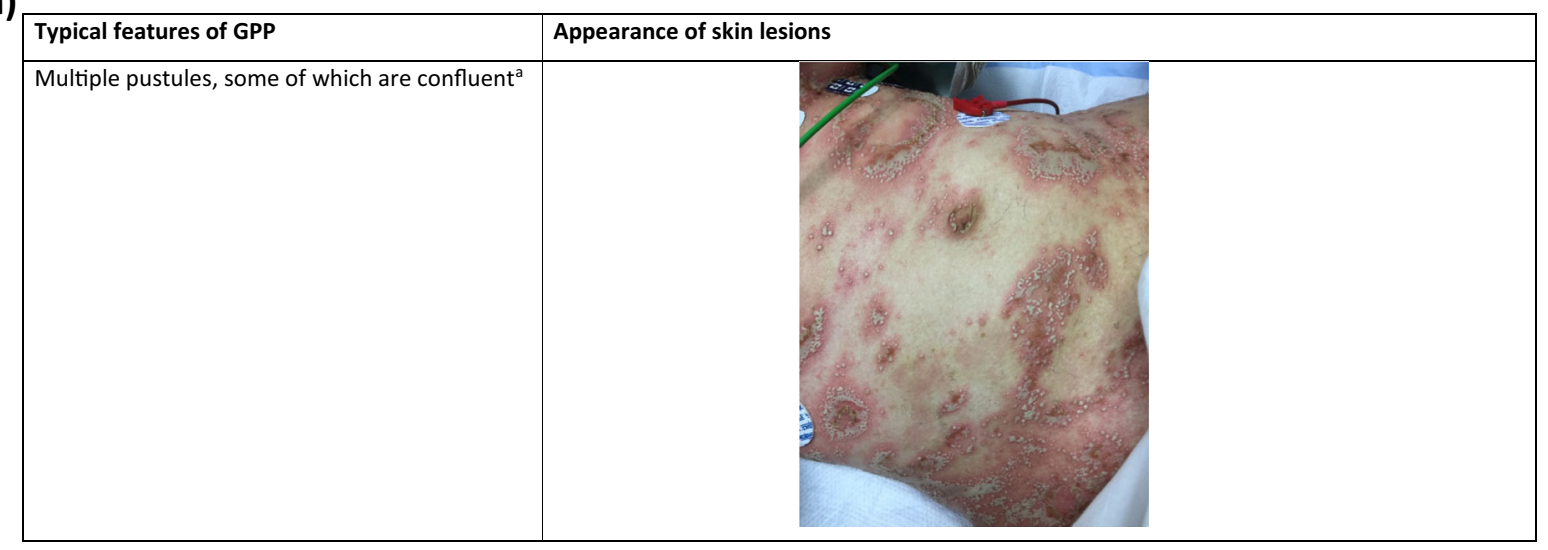

(b)

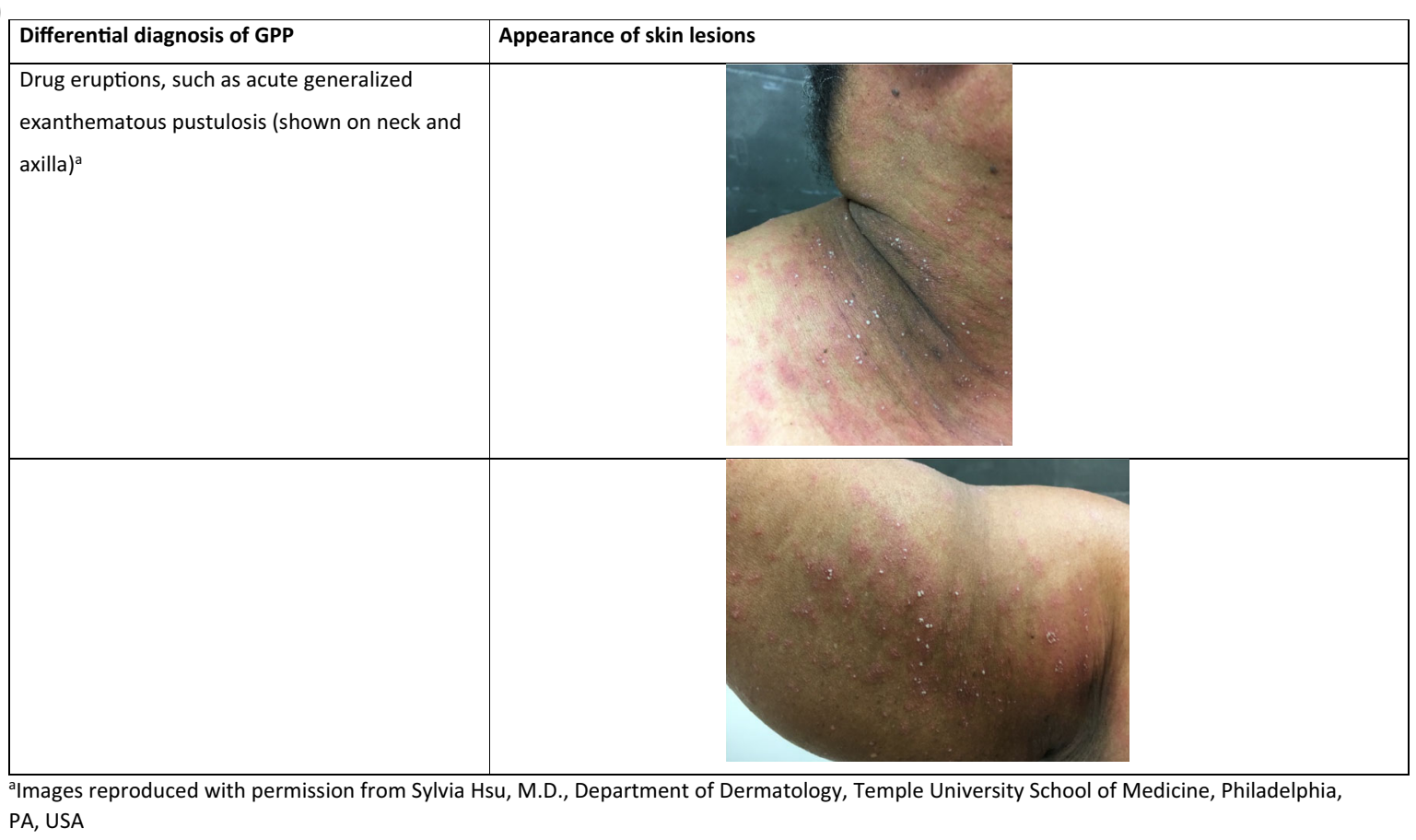

Fig. 2 Clinical features of GPP (a) and differential diagnosis (b). GPP generalized pustular psoriasis

for its receptor [34]. The IL-36RA molecule inhibits the effects of several IL-36-associated cytokines, including members of the IL- 1 cyto-

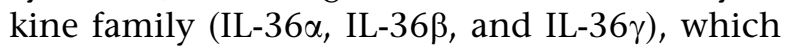
are abundantly expressed in the skin. Binding of IL-36R by an agonist cytokine in the IL-1 family results in uncontrolled activation of the transcription factor, NF- $\kappa \mathrm{B}$. This leads to activation of proinflammatory pathways via massive release of inflammatory mediators, such as
CXCL8, TNF $\alpha$, IL-1, and IL-23 from keratinocytes, macrophages, and dendritic cells $[2,33]$. In the absence of a functional IL-36RA, IL-36 is uninhibited in its ability to enhance the levels of proinflammatory mediators [1]. The IL36 receptor is found on several skin cell types and, when activated, leads to inflammation of the skin and symptoms of GPP.

Mutations or variants of CARD14, a key regulator of skin immune homeostasis, are also 
associated with GPP [41, 42]. Mutations of the AP1S3 gene have also been found in subtypes of pustular psoriasis, mainly in GPP and localized PPP (acrodermatitis continua of Hallopeau). The resulting structural and functional changes in the adaptor protein 1 family lead to deregulation of innate immune responses in the skin $[43,44]$.

\section{Treatment Options}

In general, treatment for pustular psoriasis is determined by the extent and severity of the disease. Although pustular psoriasis is distinct from plaque psoriasis, most therapies for PPP and GPP have been based on traditional treatment approaches for plaque psoriasis $[45,46]$. From the 1980s to the early 2000s, there has been a trend toward increasing use of high potency topical corticosteroids for the management of plaque psoriasis [47]. However, treatment may also include phototherapy, systemic therapies (e.g., cyclosporine, methotrexate, acitretin, apremilast), and systemic immunomodulatory agents (biologic therapies) [1]. Clinical experience suggests that biologic therapies with the greatest efficacy for plaque psoriasis are also more likely to be effective in pustular psoriasis, although clinical trial data in pustular psoriasis are limited [48]. Current classes of biologic therapies with approval from the US Food and Drug Administration and the European Medicines Agency for treatment of moderate-to-severe plaque psoriasis in the USA (as well as other countries) include four TNFa inhibitors (adalimumab, certolizumab pegol, etanercept, and infliximab), three IL-17 inhibitors (brodalumab, ixekizumab, and secukinumab), an IL-12/23 inhibitor (ustekinumab), and three IL-23 inhibitors (guselkumab, risankizumab, and tildrakizumab) [6, 48-50].

\section{Treatments for PPP}

At present, there is insufficient evidence to determine which therapies are most effective for chronic PPP [15, 48]. Current treatment options include superpotent corticosteroids (with or without occlusion), phototherapy, and systemic therapies (Fig. 3) [10, 51]. However, the use of systemic immunosuppressant agents can be associated with a slow onset of action and poor tolerability $[1,46]$.

Guselkumab, an IL-23 monoclonal antibody, is the first biologic therapy to have demonstrated moderate efficacy and a good safety profile in Japanese patients with PPP $[10,52,53]$. Ustekinumab, an inhibitor of IL-12 and IL-23, has been used in the treatment of PPP, but evidence for its efficacy is conflicting $[10,15]$. The IL-17 inhibitor secukinumab has also been evaluated for moderate-to-severe PPP, but did not demonstrate superiority versus placebo in achievement of the primary endpoint (75\% improvement from baseline in Palmoplantar Pustulosis Psoriasis Area and Severity Index [PPPASI]) [54]. Brodalumab, another IL-17 inhibitor, has been evaluated in PPP and has demonstrated either no improvement or moderate improvement in a recent case series of treated patients [55]. A small $(N=15)$, randomized, placebo-controlled, prospective study of a TNFa inhibitor, etanercept, has shown mixed results, with some patients experiencing improvements and others showing worsening PPP [56]. Apremilast, an oral phosphodiesterase 4 inhibitor, also has moderate evidence of clinical efficacy in PPP [10].

Recent trials in PPP have focused on blockade of the IL- 1 or IL-36 pathways, which have a central role in innate immunity $[10,51]$. Anakinra, a recombinant IL-1 receptor antagonist, is undergoing evaluation in patients with PPP [57]. The novel IL-36 receptor inhibitor spesolimab has been shown to be effective in a phase 1 proof-of-concept study of seven patients with GPP; it is also being evaluated in patients with PPP [58-60]. The efficacy of spesolimab in patients with GPP was demonstrated regardless of the presence of IL36RN mutation, a finding that may be of importance for patients with PPP, since this mutation occurs in only a minority of cases. The efficacy and safety of spesolimab was assessed in a placebo-controlled pilot study of 59 patients with PPP [61]. Although the primary endpoint was not met (proportion of patients achieving a $50 \%$ reduction in PPPASI score at week 16 for spesolimab vs. placebo), a post hoc subanalysis of patients with baseline PPPASI above median 


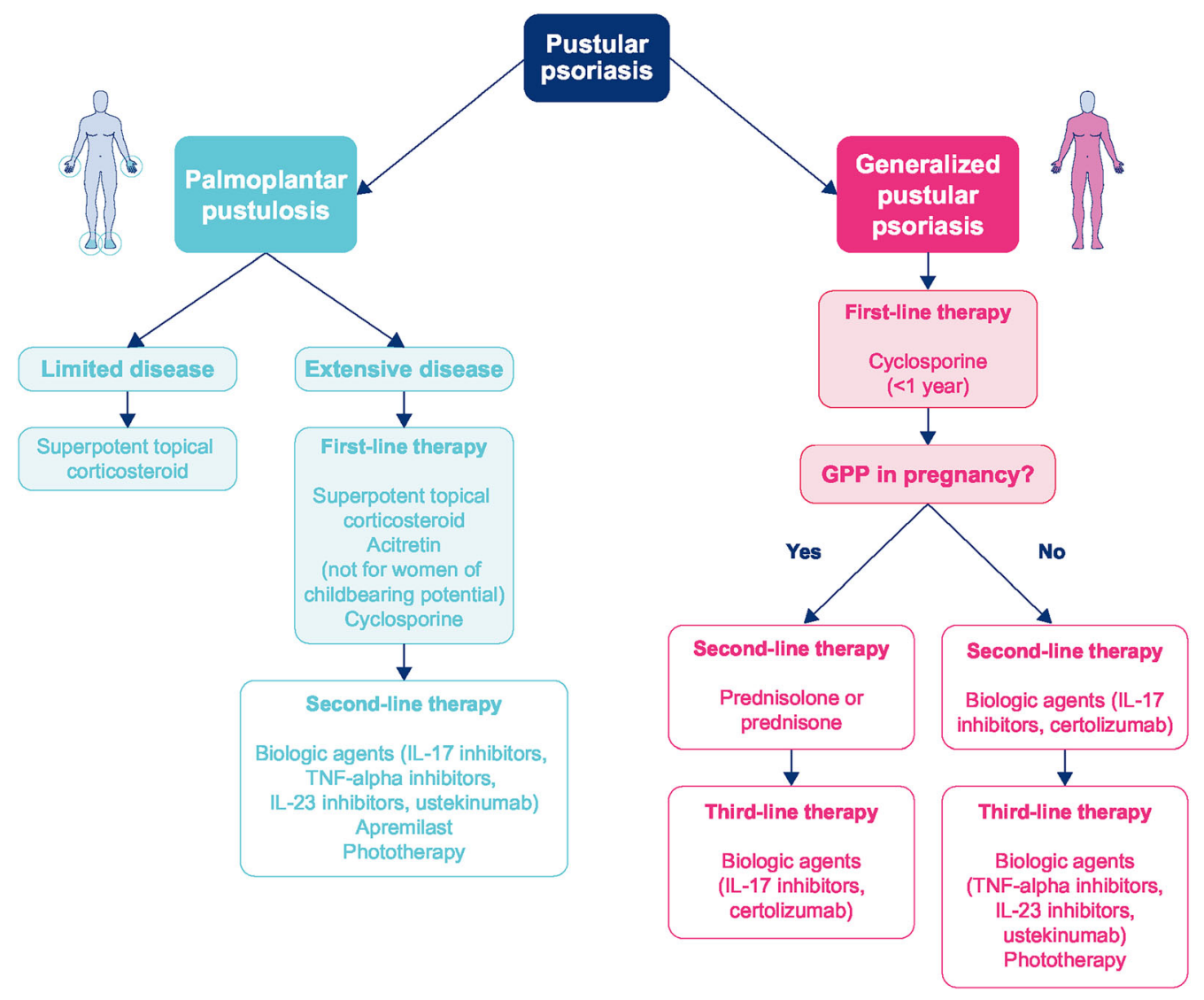

Fig. 3 Treatment algorithm for pustular psoriasis. GPP generalized pustular psoriasis, IL interleukin, $P U V A$ psoralen + ultraviolet light $\mathrm{A}, T N F$ tumor necrosis factor, $U V B$ ultraviolet $\mathrm{B}$

showed rapid improvement with spesolimab versus placebo in this subgroup; a further trial is currently under way. Another IL-36 receptor inhibitor, imsidolimab, is also under evaluation in patients with PPP [62]. Further targeted therapies are currently being investigated for use in PPP, with targets including CXCR2 (an IL-8 receptor type $B$ agent) and granulocyte colony-stimulating factor receptors, IL-12, and TNF $\alpha[10]$.

\section{Treatments for GPP}

The clinical trial evidence for GPP is more limited than for PPP because of the rarity of GPP. The spontaneously remitting pattern of acute GPP flares also makes clinical evaluation of therapies difficult [2]. As a result, there is a lack of evidence-based treatment guidelines [1], and clinicians must rely on evidence from open-label studies and case reports for guidance on efficacy in GPP [63-65]. Treatment options for patients with GPP are limited and based on existing therapies for plaque psoriasis [25]. Most therapies show weak evidence of efficacy in GPP, and some agents are not well tolerated [25]. Most patients with mild-to-moderate symptoms can experience symptom reduction with topical medications or phototherapy, although these options are unlikely to be effective for patients with more severe disease. Biologic therapies, as monotherapy or combined with other topical or systemic medications, offer additional treatment options (Fig. 3). While IL-36 inhibitors, such as spesolimab, have the potential to increase the treatment options for patients with GPP, these agents are not currently approved in the USA and are, therefore, not included in the current treatment algorithm. For the management of GPP in pregnancy, special consideration is 
required. Initial treatment for pregnant women involves cyclosporine $4-5 \mathrm{mg} / \mathrm{kg}$ of ideal body weight divided into two doses per day $[66,67]$. Second-line options include corticosteroids (such as prednisolone or prednisone, with monitoring for potential rebound exacerbation after treatment cessation) or biologic therapies (faster acting IL-17 or IL-23 inhibitors, or certolizumab, which does not cross the placenta) (Fig. 3). The associated placental insufficiency, which is potentially life-threatening to the unborn child, requires an efficacious medication that has a rapid onset of action [25].

There remains a need for an effective treatment with a rapid onset of action, and for robust clinical trial data to guide treatment decisions. Newer treatments for GPP are emerging, including IL-17 inhibitors, which have been approved for the treatment of GPP in Japan (IL-17A monoclonal antibodies: brodalumab, ixekizumab, and secukinumab) $[48,68]$. Other approved agents for GPP in Japan are IL-23 agents, guselkumab and risankizumab, and TNF $\alpha$ agents, infliximab and adalimumab $[48,68]$. Anakinra, a recombinant IL-1 receptor antagonist, has also been reported to reduce symptoms in two case series of patients with GPP and IL36RN mutations $[69,70]$.

High levels of $\mathrm{TNF} \alpha$, a cytokine that activates proinflammatory nuclear transcription factors and keratinocyte proliferation, are a key pathogenic feature of the skin inflammation that characterizes GPP [71]. First-generation biologic therapies target $\mathrm{TNF} \alpha$, with a potentially lower risk of end-organ damage compared with traditional treatments. Etanercept, a fusion protein containing human $\mathrm{TNF} \alpha$ receptor, has been shown to be moderately effective in reducing the symptoms of GPP [72], including pediatric GPP [73]. More recently, specific inhibitors of the IL-36 pathway are emerging for the management of both GPP and PPP. Of these, the IL-36 receptor inhibitor spesolimab is at the most advanced stage of development, with confirmatory trials currently under way in GPP. In a phase 1 proof-of-concept study (NCT02978690), a single infusion of spesolimab to seven patients with moderate-to-severe GPP flares was associated with rapid and sustained improvements in clinical symptoms [60]. A Generalized Pustular Psoriasis Physician Global Assessment score of 0 or 1 was achieved within 1 week and sustained to week 20 , irrespective of the presence of IL36RN mutation. Pustules were completely clear in three patients within $48 \mathrm{~h}$ after treatment, in five patients by week 1 , and in six patients by week 2 [60]. The findings of this proof-of-concept study suggest that IL-36 receptor inhibition with a single dose of spesolimab could significantly reduce the severity of GPP over a 20 -week period. Since GPP is a systemic disease, it is of interest that spesolimab was also associated with a rapid and sustained reduction in inflammation, as indicated by reductions in C-reactive protein and neutrophil levels to week 4 [60]. Another IL-36 receptor inhibitor, imsidolimab, is also being evaluated in GPP and PPP, and phase 2 trials are ongoing [74]. Further research into agents acting on the IL-36 pathway and other targeted therapies has the potential to transform the treatment of patients with pustular psoriasis for whom few effective treatment options are currently available.

\section{DISCUSSION}

\section{Clinical Management of Patients with Pustular Psoriasis}

Pustular psoriasis presents several diagnostic and management challenges to dermatologists. The less common nature of both PPP and GPP can make diagnosis problematic, and treatment decisions are difficult because of limited treatment options. Current management tends to follow treatment options for plaque psoriasis, and guidelines on the management of psoriasis with biologic therapies have recently been published [6, 7]. However, specific guidelines for PPP and GPP are lacking, and there remains an important need for a treatment with high efficacy and rapid onset of action for both forms of pustular psoriasis.

Recent clinical trials of targeted therapies for GPP have shown encouraging results for the management of the debilitating skin diseases within the pustular psoriasis spectrum, and 
offer the potential to set a new standard in patient care with the possibility of rapid and effective treatment. These emerging drugs for pustular psoriasis offer lower toxicities than existing therapies. The development of new evidence-based guidelines for the management of pustular psoriasis is anticipated for North America and Europe, with the potential to assist clinicians with management decisions, leading to further significant and important improvements in outcomes for patients with both forms of pustular psoriasis.

\section{ACKNOWLEDGEMENTS}

Funding. Boehringer Ingelheim Pharmaceuticals, Inc. (BIPI) funded all publication fees. BIPI was given the opportunity to review the manuscript for medical and scientific accuracy as well as intellectual property considerations.

Medical Writing Assistance. Writing support was provided by Jennifer Garrett, MBBS, of Elevate Scientific Solutions, which was contracted and funded by BIPI.

Authorship. All named authors meet the International Committee of Medical Journal Editors (ICMJE) criteria for authorship for this article, take responsibility for the integrity of the work as a whole, and have given their approval for this version to be published. The authors received no direct compensation related to the development of the manuscript.

Author Contributions. All authors contributed to the conception of the review. Jennifer Garrett performed the initial literature search; all authors contributed to further literature searches and the literature analysis. The first draft of the manuscript was written by Jennifer Garrett and all authors commented on and critically revised subsequent drafts. All authors read and approved the final manuscript. Sylvia Hsu provided the images.

Disclosures. Alan Menter reports receiving support from Abbott Laboratories, AbbVie,
Amgen, Boehringer Ingelheim, Celgene, Eli Lilly, Janssen Biotech, Inc., LEO Pharma, Merck, Novartis, SunPharma, and UCB Pharma. Abby S. Van Voorhees reports receiving support from AbbVie, Allergan, Amgen, BMS, Boehringer Ingelheim, Celgene, Corrona, Dermira, Merck, Novartis, Pfizer, UCB Pharma, and Valeant. Sylvia Hsu reports receiving support from Amgen, Celgene, Corrona, Eli Lilly, and Novartis in 2020.

Compliance with Ethics Guidelines. This article is based on previously conducted studies and does not contain any new studies with human participants or animals performed by any of the authors.

Data Availability. Data sharing is not applicable to this article as no data sets were generated or analyzed during the current study.

Open Access. This article is licensed under a Creative Commons Attribution-NonCommercial 4.0 International License, which permits any non-commercial use, sharing, adaptation, distribution and reproduction in any medium or format, as long as you give appropriate credit to the original author(s) and the source, provide a link to the Creative Commons licence, and indicate if changes were made. The images or other third party material in this article are included in the article's Creative Commons licence, unless indicated otherwise in a credit line to the material. If material is not included in the article's Creative Commons licence and your intended use is not permitted by statutory regulation or exceeds the permitted use, you will need to obtain permission directly from the copyright holder. To view a copy of this licence, visit http://creativecommons.org/licenses/by$\mathrm{nc} / 4.0 /$.

\section{REFERENCES}

1. Benjegerdes KE, Hyde K, Kivelevitch D, Mansouri B. Pustular psoriasis: pathophysiology and current treatment perspectives. Psoriasis (Auckl). 2016;6: 131-44. 
2. Bachelez H. Pustular psoriasis: the dawn of a new era. Acta Derm Venereol. 2020;100:adv00034.

3. Ciccarelli F, De Martinis M, Sirufo MM, Ginaldi L. Psoriasis induced by anti-tumor necrosis factor alpha agents: a comprehensive review of the literature. Acta Dermatovenerol Croat. 2016;24:169-74.

4. Mossner R, Pinter A. Paradoxical palmoplantar pustulosis induced by secukinumab and brodalumab: a report of three cases. Eur J Dermatol. 2020;30:177-8.

5. Satoh M, Yamamoto T. Secukinumab-induced palmar vesiculopustular eruption in a patient with psoriasis. Dermatol Ther. 2020;33:e13332.

6. Menter A, Strober BE, Kaplan DH, et al. Joint AADNPF guidelines of care for the management and treatment of psoriasis with biologics. J Am Acad Dermatol. 2019;80:1029-72.

7. Smith $\mathrm{CH}$, Yiu ZZN, Bale T, et al. British Association of Dermatologists guidelines for biologic therapy for psoriasis 2020: a rapid update. Br J Dermatol. 2020;183:628-37.

8. Kharawala S, Golembesky AK, Bohn RL, Esser D. The clinical, humanistic, and economic burden of palmoplantar pustulosis: a structured review. Expert Rev Clin Immunol. 2020;16:253-66.

9. Kubota K, Kamijima Y, Sato T, et al. Epidemiology of psoriasis and palmoplantar pustulosis: a nationwide study using the Japanese national claims database. BMJ Open. 2015;5:e006450.

10. Misiak-Galazka M, Zozula J, Rudnicka L. Palmoplantar pustulosis: recent advances in etiopathogenesis and emerging treatments. Am J Clin Dermatol. 2020;21:355-70.

11. Navarini AA, Burden AD, Capon F, et al. European consensus statement on phenotypes of pustular psoriasis. J Eur Acad Dermatol Venereol. 2017;31: 1792-9.

12. Twelves S, Mostafa A, Dand N, et al. Clinical and genetic differences between pustular psoriasis subtypes. J Allergy Clin Immunol. 2019;143:1021-6.

13. Misiak-Galazka M, Wolska H, Galazka A, Kwiek B, Rudnicka L. General characteristics and comorbidities in patients with palmoplantar pustulosis. Acta Dermatovenerol Croat. 2018;26:109-18.

14. Kouno M, Nishiyama A, Minabe M, et al. Retrospective analysis of the clinical response of palmoplantar pustulosis after dental infection control and dental metal removal. J Dermatol. 2017;44:695-8.
15. Obeid G, Do G, Kirby L, et al. Interventions for chronic palmoplantar pustulosis. Cochrane Database Syst Rev. 2020;1:CD011628.

16. Ko JM, Gottlieb $\mathrm{AB}$, Kerbleski JF. Induction and exacerbation of psoriasis with TNF-blockade therapy: a review and analysis of 127 cases. J Dermatol Treat. 2009;20:100-8.

17. Garcovich S, De Simone C, Genovese G, et al. Paradoxical skin reactions to biologics in patients with rheumatologic disorders. Front Pharmacol. 2019;10:282.

18. Stoffel E, Maier H, Riedl E, et al. Analysis of antitumour necrosis factor-induced skin lesions reveals strong $\mathrm{T}$ helper 1 activation with some distinct immunological characteristics. $\mathrm{Br} \mathrm{J}$ Dermatol. 2018;178:1151-62.

19. Mossner R, Frambach Y, Wilsmann-Theis D, et al. Palmoplantar pustular psoriasis is associated with missense variants in CARD14, but not with loss-offunction mutations in IL36RN in European patients. J Investig Dermatol. 2015;135:2538-41.

20. Murakami M, Terui T. Palmoplantar pustulosis: current understanding of disease definition and pathomechanism. J Dermatol Sci. 2020;98:13-9.

21. Mahil SK, Catapano M, Di Meglio P, et al. An analysis of IL-36 signature genes and individuals with IL1RL2 knockout mutations validates IL-36 as a psoriasis therapeutic target. Sci Transl Med. 2017;9:eaan2514. https://doi.org/10.1126/ scitranslmed.aan2514.

22. Bissonnette R, Fuentes-Duculan J, Mashiko S, et al. Palmoplantar pustular psoriasis (PPPP) is characterized by activation of the IL-17A pathway. J Dermatol Sci. 2017;85:20-6.

23. Augey F, Renaudier P, Nicolas JF. Generalized pustular psoriasis (Zumbusch): a French epidemiological survey. Eur J Dermatol. 2006;16:669-73.

24. Ohkawara A, Yasuda H, Kobayashi H, et al. Generalized pustular psoriasis in Japan: two distinct groups formed by differences in symptoms and genetic background. Acta Derm Venereol. 1996;76: $68-71$.

25. Fujita H, Terui T, Hayama $\mathrm{K}$, et al. Japanese guidelines for the management and treatment of generalized pustular psoriasis: the new pathogenesis and treatment of GPP. J Dermatol. 2018;45:1235-70.

26. Takeichi T, Akiyama M. Generalized pustular psoriasis: clinical management and update on autoinflammatory aspects. Am J Clin Dermatol. 2020;21: 227-36. 
27. Umezawa Y, Ozawa A, Kawasima T, et al. Therapeutic guidelines for the treatment of generalized pustular psoriasis (GPP) based on a proposed classification of disease severity. Arch Dermatol Res. 2003;295(Suppl 1):S43-54.

28. Sidoroff A, Halevy S, Bavinck JN, Vaillant L, Roujeau JC. Acute generalized exanthematous pustulosis (AGEP)-a clinical reaction pattern. J Cutan Pathol. 2001;28:113-9.

29. Brenner M, Molin S, Ruebsam K, et al. Generalized pustular psoriasis induced by systemic glucocorticosteroids: four cases and recommendations for treatment. Br J Dermatol. 2009;161:964-6.

30. Vena GA, Cassano N, Bellia G, Colombo D. Psoriasis in pregnancy: challenges and solutions. Psoriasis (Auckl). 2015;5:83-95.

31. Sbidian E, Madrange M, Viguier M, et al. Respiratory virus infection triggers acute psoriasis flares across different clinical subtypes and genetic backgrounds. Br J Dermatol. 2019;181:1304-6.

32. Furue K, Yamamura K, Tsuji G, et al. Highlighting interleukin-36 signalling in plaque psoriasis and pustular psoriasis. Acta Derm Venereol. 2018;98: 5-13.

33. Johnston A, Xing X, Wolterink L, et al. IL-1 and IL36 are dominant cytokines in generalized pustular psoriasis. J Allergy Clin Immunol. 2017;140: 109-20.

34. Marrakchi S, Guigue P, Renshaw BR, et al. Interleukin-36-receptor antagonist deficiency and generalized pustular psoriasis. N Engl J Med. 2011;365: 620-8.

35. Gooderham MJ, Van Voorhees AS, Lebwohl MG. An update on generalized pustular psoriasis. Expert Rev Clin Immunol. 2019;15:907-19.

36. Sugiura K, Takemoto A, Yamaguchi M, et al. The majority of generalized pustular psoriasis without psoriasis vulgaris is caused by deficiency of interleukin-36 receptor antagonist. J Investig Dermatol. 2013;133:2514-21.

37. Hussain S, Berki DM, Choon SE, et al. IL36RN mutations define a severe autoinflammatory phenotype of generalized pustular psoriasis. J Allergy Clin Immunol. 2015;135:1067-70 (e9).

38. Korber A, Mossner R, Renner R, et al. Mutations in IL36RN in patients with generalized pustular psoriasis. J Investig Dermatol. 2013;133:2634-7.

39. Li Z, Yang Q, Wang S. Genetic polymorphism of IL36RN in Han patients with generalized pustular psoriasis in Sichuan region of China: a case-control study. Medicine (Baltimore). 2018;97:e11741.

40. Takeichi T, Togawa Y, Okuno Y, et al. A newly revealed IL36RN mutation in sibling cases complements our IL36RN mutation statistics for generalized pustular psoriasis. J Dermatol Sci. 2017;85: 58-60.

41. Berki DM, Liu L, Choon SE, et al. Activating CARD14 mutations are associated with generalized pustular psoriasis but rarely account for familial recurrence in psoriasis vulgaris. J Investig Dermatol. 2015;135:2964-70.

42. Manils J, Webb LV, Howes A, et al. CARD14(E138A) signalling in keratinocytes induces TNF-dependent skin and systemic inflammation. Elife. 2020;9: e56720. https://doi.org/10.7554/eLife.56720.

43. Mahil SK, Twelves S, Farkas K, et al. AP1S3 mutations cause skin autoinflammation by disrupting keratinocyte autophagy and up-regulating IL-36 production. J Investig Dermatol. 2016;136:2251-9.

44. Setta-Kaffetzi N, Simpson MA, Navarini AA, et al. AP1S3 mutations are associated with pustular psoriasis and impaired Toll-like receptor 3 trafficking. Am J Hum Genet. 2014;94:790-7.

45. Bachelez H. Pustular psoriasis and related pustular skin diseases. Br J Dermatol. 2018;178:614-8.

46. Robinson A, Van Voorhees AS, Hsu S, et al. Treatment of pustular psoriasis: from the Medical Board of the National Psoriasis Foundation. J Am Acad Dermatol. 2012;67:279-88.

47. Strowd LC, Yentzer BA, Fleischer AB Jr, Feldman SR. Increasing use of more potent treatments for psoriasis. J Am Acad Dermatol. 2009;60:478-81.

48. Saeki H, Terui T, Morita A, et al. Japanese guidance for use of biologics for psoriasis (the 2019 version). J Dermatol. 2020;47:201-22.

49. European Medicines Agency. Medicines. https:// www.ema.europa.eu/en/medicines. Accessed 24 Nov 2020.

50. National Institute of Clinical Excellence. Systemic biological therapy for psoriasis. https://pathways. nice.org.uk/pathways/psoriasis\#path=view\%3A/ pathways/psoriasis/systemic-biological-therapy-forpsoriasis.xml\&content=view-index. Accessed 24 Nov 2020.

51. Freitas E, Rodrigues MA, Torres T. Diagnosis, screening and treatment of patients with palmoplantar pustulosis (PPP): a review of current practices and recommendations. Clin Cosmet Investig Dermatol. 2020;13:561-78. 
52. Sano S, Kubo H, Morishima H, et al. Guselkumab, a human interleukin-23 monoclonal antibody in Japanese patients with generalized pustular psoriasis and erythrodermic psoriasis: efficacy and safety analyses of a 52-week, phase 3, multicenter, openlabel study. J Dermatol. 2018;45:529-39.

53. Terui T, Kobayashi S, Okubo Y, et al. Efficacy and safety of guselkumab in Japanese patients with palmoplantar pustulosis: a phase 3 randomized clinical trial. JAMA Dermatol. 2019;155:1153-61.

54. Mrowietz U, Bachelez H, Burden AD, et al. Secukinumab for moderate-to-severe palmoplantar pustular psoriasis: results of the 2PRECISE study. J Am Acad Dermatol. 2019;80:1344-52.

55. Pinter A, Wilsmann-Theis D, Peitsch WK, Mossner R. Interleukin-17 receptor A blockade with brodalumab in palmoplantar pustular psoriasis: report on four cases. J Dermatol. 2019;46:426-30.

56. Bissonnette R, Poulin Y, Bolduc C, et al. Etanercept in the treatment of palmoplantar pustulosis. J Drugs Dermatol. 2008;7:940-6.

57. Cornelius V, Wilson R, Cro S, et al. A small population, randomised, placebo-controlled trial to determine the efficacy of anakinra in the treatment of pustular psoriasis: study protocol for the APRICOT trial. Trials. 2018;19:465.

58. Clinicaltrials.gov. Initial dosing of BI 655130 in palmoplantar pustulosis patients. https:// clinicaltrials.gov/ct2/show/nct03135548. Accessed 15 Oct 2020.

59. Clinicaltrials.gov. A study to test how effective and safe different doses of BI 655130 are in patients with a moderate to severe form of the skin disease palmoplantar pustulosis. https://clinicaltrials.gov/ct2/ show/NCT04015518. Accessed 15 Oct 2020.

60. Bachelez H, Choon SE, Marrakchi S, et al. Inhibition of the interleukin-36 pathway for the treatment of generalized pustular psoriasis. $\mathrm{N}$ Engl J Med. 2019;380:981-3.

61. Mrowietz U, Burden AD, Pinter A, Reich K, Schäkel K, Baum P, Datsenko Y, Deng H, Padula SJ, Thoma C, Bissonnette R. Spesolimab, an anti-interleukin36 receptor antibody, in patients with palmoplantar pustulosis: Results of a phase IIa, multicenter, double-blind, randomized, placebo-controlled pilot study. Dermatol Ther (Heidelb). 2021;11:571-85.

62. Clinicaltrials.gov. A study to evaluate the efficacy and safety of ANB019 in subjects with palmoplantar pustulosis (PPP). https://clinicaltrials.gov/ct2/ show/nct03633396. Accessed 15 Oct 2020.
63. Imafuku S, Honma M, Okubo Y, et al. Efficacy and safety of secukinumab in patients with generalized pustular psoriasis: a 52-week analysis from phase III open-label multicenter Japanese study. J Dermatol. 2016;43:1011-7.

64. Saeki H, Nakagawa H, Nakajo K, et al. Efficacy and safety of ixekizumab treatment for Japanese patients with moderate to severe plaque psoriasis, erythrodermic psoriasis and generalized pustular psoriasis: results from a 52-week, open-label, phase 3 study (UNCOVER-J). J Dermatol. 2017;44:355-62.

65. Yamasaki K, Nakagawa H, Kubo Y, Ootaki K, Japanese Brodalumab Study Group. Efficacy and safety of brodalumab in patients with generalized pustular psoriasis and psoriatic erythroderma: results from a 52-week, open-label study. Br J Dermatol. 2017;176: 741-51.

66. Amor KT, Ryan C, Menter A. The use of cyclosporine in dermatology: part I. J Am Acad Dermatol. 2010;63:925-46 (quiz 47-8).

67. Ryan C, Amor KT, Menter A. The use of cyclosporine in dermatology: part II. J Am Acad Dermatol. 2010;63:949-72 (quiz 73-4).

68. Wang WM, Jin HZ. Biologics in the treatment of pustular psoriasis. Expert Opin Drug Saf. 2020;19(8):969-980.

69. Viguier M, Guigue P, Pages C, Smahi A, Bachelez H. Successful treatment of generalized pustular psoriasis with the interleukin-1-receptor antagonist anakinra: lack of correlation with IL1RN mutations. Ann Intern Med. 2010;153:66-7.

70. Huffmeier U, Watzold M, Mohr J, Schon MP, Mossner R. Successful therapy with anakinra in a patient with generalized pustular psoriasis carrying IL36RN mutations. Br J Dermatol. 2014;170:202-4.

71. Schottelius AJ, Moldawer LL, Dinarello CA, et al. Biology of tumor necrosis factor-alpha - implications for psoriasis. Exp Dermatol. 2004;13:193-222.

72. Esposito M, Mazzotta A, Casciello C, Chimenti S. Etanercept at different dosages in the treatment of generalized pustular psoriasis: a case series. Dermatology. 2008;216:355-60.

73. Saikaly SK, Mattes M. Biologics and pediatric generalized pustular psoriasis: an emerging therapeutic trend. Cureus. 2016;8:652.

74. AnaptysBio. AnaptysBio announces orphan drug designation of imsidolimab for treatment of generalized pustular psoriasis. http://ir.anaptysbio. com/news-releases/news-release-details/anaptysbioannounces-orphan-drug-designation-imsidolimab. Accessed 19 Oct 2020. 